\title{
On the Order Form of the Fundamental Theorems of Asset Pricing
}

\author{
Christos E. Kountzakis \\ ${ }^{1}$ Department of Mathematics, University of the Aegean, Samos, Greece \\ ${ }^{2}$ Faculty of Mathematics - Group of Finance, University of Vienna, Vienna, Austria \\ Email: chr koun@aegean.gr
}

Received 3 May 2014; revised 4 June 2014; accepted 26 June 2014

Copyright (C) 2014 by author and Scientific Research Publishing Inc.

This work is licensed under the Creative Commons Attribution International License (CC BY).

http://creativecommons.org/licenses/by/4.0/

c) (i) Open Access

\begin{abstract}
In this article, we provide an order-form of the First and the Second Fundamental Theorem of Asset Pricing both in the one-period market model for a finite and infinite state-space and in the case of multi-period model for a finite state-space and a finite time-horizon. The space of the financial positions is supposed to be a Banach lattice. We also prove relevant results in the case where the space of the financial positions is not ordered by a lattice cone.
\end{abstract}

\section{Keywords}

Strictly Positive Extension, Positive Projection, Sublattice, Complete Market, Incomplete Market

\section{Some Remarks on Previous Work about the Fundamental Asset Pricing Theorems}

The First Fundamental Theorem of Asset Pricing states that the absence of arbitrage for a stochastic process $X$ is equivalent to the existence of an equivalent martingale measure for $X$. It was shown in [1] that for a locally bounded $\mathbb{R}^{d}$-valued semi-martingale $X$ the condition of No Free Lunch with Vanishing Risk is equivalent to the existence of an equivalent local martingale measure for the process $X$. It was proved in [2] that the local boundedness assumption on $X$ may be dropped under the notion of equivalent $\sigma$-martingale measure. The work [3], also discussed in [4], is still essential in this topic and actually this work's results rely on what Kreps established as the viable market model consisted by an incomplete market and a linear price system on it. In the present work we are going to resolve the so-called Strictly Positive Extension Property from the financial aspect. The presence of heavy-tails in continuous time models and the possible change of frame from $L^{p}$ spaces to Orlicz spaces in order to fit the modelling requirements, oblige us to search for more general versions of the two FTAPs, mostly relied on the geometry of these spaces. Recently, in [5], a Fundamental Theorem of Asset Pric- 
ing and a Super-Replication Theorem in a model-independent framework are both proposed. But these theorems are proved in the setting of finite, discrete time and a market consisting of a risky asset $S$, as well as options written on this risky asset, too. Notions like the one of the strictly positive projection or that of the filtration are alike the ones met in [6]. A difference between our notion of strictly positive projection and the equivalent notion in [6] is that ours is weaker. That's because if $x>0$ implies $P x>0$, this implies $P x=0 \Rightarrow x=0$, because if $x>0$, it would be $P x>0$. An important difference between the article of Troitsky and ours is that we extend the framework of Definitions so as to include cases of non-discrete time spaces. Another one is that we apply these notions in order to provide a new version of the two FTAP, while in [6] an important ordered -space theory of martingales in Banach lattices is developed. Finally, markets subspaces are taken to be sublattices because of the fact that we may include layers of call and put options written on an initial market space, as we remarked in [7]. The present paper is organized as follows: First, we provide some useful notions and definitions and examples for them, as well. Next, we prove the Order Form of the FTAP in the Banach-lattice case and in the next sections we provide the analog of these results in the finite-models case. We also explain the application of our results on the Black-Scholes-Merton model. We also compare them to the Example developed in [4]. The case of non-lattice cones is examined in the last section of the paper, in relation with the classes of reflexive and strongly reflexive cones, mentioned in [8]. The role of the existence of an unconditional basic sequence in a Banach space is also quoted in this section independently from the results provided in [8], as an important condition for the extraction of results concerning FTAP. This condition is not irrelevant to ([9], Th. 1.1), about Lindelöf Properties of weak topology, but here it mainly concerns the construction of a Strictly Positive Projection Operator. On the other side, in the paper [10] ideals of $L^{0}(\mu)$ are used in order to deduce an FTAP-like result ([10], Lem. 1), while our results refer to sublattices.

\section{Useful Notions and Preliminaries}

We consider two periods of time ( 0 and 1 ) and a non-empty set of states of the world $\Omega$ which is supposed to be an infinite set. The true state $\omega \in \Omega$ that the investors face is contained in some $A \in \mathcal{F}$, where $\mathcal{F}$ is some $\sigma$-algebra of subsets of $\Omega$ which gives the information about the states that may occur at time-period 1 . A financial position is a $\mathcal{F}$-measurable random variable $x: \Omega \rightarrow \mathbb{R}$. This random variable is the profile of this position at time-period 1 . We suppose that the probability of any state of the world to occur is given by a probability measure $\mu: \mathcal{F} \rightarrow[0,1]$. The financial positions are supposed to lie in some subspace $E$ of $L^{0}(\Omega, \mathcal{F}, \mu)$, being a Banach lattice.

Definition 1 An incomplete market in $E$ is some sublattice $M$ of $E$. A complete market in $E$ is some sublattice $M$ of $E$, such that $\bar{M}=E$.

It is well-known that we define the positive cone $F_{+}$of a subspace $F$ of an ordered vector space to be the set $F_{+}=F \cap E_{+}$, where $E_{+}$denotes the positive cone of $E$.

Definition 2 A positive projection $P: E \rightarrow F$ is a projection, which maps each element of $E$ to some element of its subspace $F$, such that $P\left(E_{+}\right) \subseteq F_{+}$. A positive projection is called strictly positive, if $P(x) \in F_{+}$, $P(x)=0 \Leftrightarrow x \in E_{+}, x=0$.

We also recall the notion of random field.

Definition 3 A random field is a map $X: \mathcal{T} \times \Omega \rightarrow E$ where $E$ is a Bananch lattice, $\mathcal{T}$ is a topological space and $X_{t}(\omega) \in E$, for any $t \in \mathcal{T}$. Such a random field $X$ is called associated to the pair $(\mathcal{T}, E)$.

We also may provide the notion of the filtration in the frame of random fields:

Definition 4 A filtration associated to the pair $(\mathcal{T}, E)$ is a net of projections $\left(P_{a}\right)_{a \in A}$, where $P_{a}: E \rightarrow E_{a}$, where $E_{a}$ is a sublattice of $E$ and if $b \succeq a, P_{a} P_{b}=P_{a}$. A is a directed set, by some binary relation $\succeq$, called direction.

Definition 5 A binary relation $\succeq$ on $A$ is called direction on $A$, if it is reflexive and transitive on $A$, while for any $a, b \in A$ there is $a \quad c \in A$, such that $c \succeq a, b$.

Definition 6 If $b \succeq a$ and $b \neq a$, this is denoted by $b \succ a, a, b \in A$.

Definition 7 A filtration $\left(P_{a}\right)_{a \in A}$ is called strictly positive if $P_{a}(x)=0, x \in E_{+} \Leftrightarrow x=0, a \in A$.

We also give the definition of the adapted random field under this frame.

Definition 8 A random field $X: A \times \Omega \rightarrow E$, where $A \subseteq \mathcal{T}$ is called adapted to the filtration $\left(P_{a}\right)_{a \in A}$, being associated to the pair $(\mathcal{T}, E)$ if $X_{a} \in E_{a}$ for any $a \in A$, where $A$ is a directed subset of $\mathcal{T}$ by some 
binary relation $\succeq$, which is reflexive, transitive and every pair has an upper bound.

Definition 9 A random field $X: A \times \Omega \rightarrow E$, where $A \subseteq \mathcal{T}$, has the Martingale Property if it is adapted to a filtration $\left(P_{a}\right)_{a \in A}$, being associated to the pair $(\mathcal{T}, E)$, while $P_{a} X_{b}=X_{a}, b \succeq a$.

Definition $10 A$ random field $X: A \times \Omega \rightarrow E$, where $A \subseteq \mathcal{T}$, has the Strictly Positive Martingale Property if it is adapted to a filtration $\left(P_{a}\right)_{a \in A}$, being associated to the pair $(\mathcal{T}, E)$, it has the Martingale Property, while the filtration $\left(P_{a}\right)_{a \in A}$ is consisted by strictly positive projections.

We give some examples for the previously mentioned notions.

Example 11 If $\mathcal{A}$ is a sub-algebra of the $\sigma$-algebra $\mathcal{F}$ of $\Omega$, then since $L^{p}(\Omega, \mathcal{A}, \mu)$ is a sublattice of $L^{p}(\Omega, \mathcal{F}, \mu), 1 \leq p<\infty$, then $L^{p}(\Omega, \mathcal{A}, \mu)$ is an incomplete market of financial positions in $L^{p}(\Omega, \mathcal{F}, \mu)$, $1 \leq p<\infty$.

Example 12 The subspace of partially linear functions $M$ in the space $C[0,1]$ is a complete market in $C[0,1]$, due to the Stone-Weierstrass Theorem. We notice that the partially linear functions defined on $[0,1]$ is actually the sublattice generated by the bi-set of functions $\left\{\phi_{1}, \phi_{2}\right\}$, where $\phi_{1}(t)=1, t \in[0,1], \phi_{2}(t)=t, t \in[0,1]$. We notice that in this case, the span of this bi-set is a lattice-subspace of $C[0,1]$ (see also [11]).

Example 13 A finite-dimensional sublattice $M$ of $C[0,1]$ is an incomplete market in $C[0,1]$. As a latticesubspace, it actually has a positive basis with nodes ([11], Pr. 2.2), hence the equivalent positive projection $P_{M}: C[0,1] \rightarrow M$ is defined as follows:

$$
P_{M}(x)(t)=\sum_{i=1}^{n} \frac{x\left(t_{i}\right)}{b\left(t_{i}\right)} b_{i}(t), t \in[0,1],
$$

where $n=\operatorname{dim} M$ and $t_{1}, t_{2}, \cdots, t_{n} \in[0,1]$ are the nodes of the positive basis of $M$.

Example 14 A sequence of sublattices $\left(M_{n}\right)_{n \in \mathbb{N}}$ of $C[0,1]$ characterized by increasing non-terminal parts of the sequence

$$
\left\{t_{1}, t_{2}, \cdots, t_{n}, t_{n+1}, \cdots\right\} \subseteq[0,1],
$$

which has different terms in the sense $t_{n} \neq t_{m}, n \neq m$ is a filtration of $C[0,1]$, since $t_{1}$ is the node for the onedimensional subspace $M_{1},\left\{t_{1}, t_{2}\right\}$ is the set of nodes of the positive basis of $M_{2}$ and so on.

Example 15 An increasing net of sub- $\sigma$-algebras $\left(\mathcal{F}_{a}\right)_{a \in A}$ of $\Omega$, being a non-empty set, where $A$ is a nonempty directed set, induces as it is well-known the existence of a filtration in $L^{1}(\Omega, \mathcal{F}, \mu)$, where $(\Omega, \mathcal{F}, \mu)$ is supposed to be a probability space associated to the measurable space $(\Omega, \mathcal{F})$. The relevant net of sublattices is:

$$
\left(L^{1}(\Omega, \mathcal{F}, \mu)\right)_{a \in A} .
$$

A may denote a set of cardinals, where if we start from a certain cardinal number $a$, then the cardinality of $\sigma$-algebra $\mathcal{F}_{a}$ as a class of objects is at most equal to $2^{a}$ and it is surely greater than $a+1$.

Example 16 The filtration of the Example 14 is not strictly positive. This holds because if we pick a sublattice $M_{n_{1}}$ whose positive basis' nodes is the set:

$$
\left\{t_{1}, t_{2}, \cdots, t_{n_{1}}\right\} \subseteq[0,1]
$$

If $x\left(t_{i}\right)=0, i=1,2, \cdots, n_{1}$, this does not imply $x=0$ if $x \geq 0$. For example, $x(t)=\prod_{i=1}^{n_{1}}\left(t-t_{i}\right)^{2} \geq 0$, but $x\left(t_{i}\right)=0, i=1,2, \cdots, n_{1}$.

Example 17 If $E$ is a Banach lattice with order continuous norm and $B$ is a projection band, namely $E=B \oplus B^{d}$, then $B$ is norm-closed. The projection $P_{B}: E \rightarrow B$ is strictly positive since it is positive and $P_{B}(x)=0, x \in E_{+}$, implies that since $x=x_{1}+x_{2}$, where $x_{1} \in B$ and $x_{2} \in B^{d}, x_{1}=0 . x_{2} \wedge 0=0$ and $x_{2} \geq 0$, hence $x_{2}=0$ and finally $x=0$. The same situation is valid for Kantorovich-Banach spaces (or else KB-spaces), in which $E^{* *}=E \oplus E^{d}$. Such examples of spaces are reflexive Banach lattices like $L^{p}(\Omega, \mathcal{F}, \mu), 1<p<\infty$ and $A L$-spaces.

Example 18 Let us consider a Banach lattice $E$ which has a Schauder basis:

$$
\left\{e_{1}, e_{2}, \cdots, e_{n}, \cdots\right\}, n \in \mathbb{N},
$$


which is moreover a positive basis. Also, suppose that:

$$
M_{n}=P_{n} E=\left[e_{1}, e_{2}, \cdots, e_{n}\right], n \in \mathbb{N},
$$

are finite-dimensional sublattices of $E$. Then,

$$
\left(P_{n}\right)_{n \in \mathbb{N}}
$$

is a filtration, because $P_{n} P_{m}=P_{n}, m \leq n$ and $P_{n} x=0 \Leftrightarrow x=0, x \in E_{+}$, since $\left\{e_{1}, e_{2}, \cdots, e_{n}\right\}$ is a positive basis of $M_{n}$ itself. This is the case for $E=c_{0}, \ell^{1}, \ell^{p}, 1<p<\infty$.

\section{Order Versions for the Fundamental Theorems of Asset Pricing}

In the proof of the two next Theorems we use the following:

Lemma 19 A positive projection $P: E \rightarrow M$, where $E$ is a Banach lattice and $M$ is a positive sublattice of it, is a continuous operator.

Proof: Obvious, because every positive operator from a Banach lattice into to a locally solid Riesz space, is continuous.

Theorem 20 (Order 1st Fundamental Theorem of Asset Pricing) Let $E$ be a Banach lattice and $M$ be a sublattice of $E$. If $M$ admits a strictly positive projection, then every strictly positive and continuous functional $f: M \rightarrow \mathbb{R}$, admits a strictly positive, continuous extension on $E$. Also, if $E$ is a Banach lattice and $M$ is a sublattice of $E$ such that every strictly positive and continuous functional $f: M \rightarrow \mathbb{R}$, admits a strictly positive, continuous extension on $E$, then $M$ admits a strictly positive projection.

Proof: The adjoint operator of the strictly positive projection $P: E \rightarrow M$ is an injection. Hence $P^{*}(f): E \rightarrow \mathbb{R}$ is a continuous, strictly positive functional of $E$. This is due to the duality:

$$
\left\langle x, P^{*}(f)\right\rangle=\langle P(x), f\rangle, x \in E .
$$

For the proof of the opposite, we have the following: We define the projection $P_{M}: E \rightarrow M$ as follows. $P_{M}(x)=x, x \in M, P_{M}(x)=0, x \notin M . P_{M}$ is a positive operator from a Banach lattice into a locally solid Riesz space. Hence it is continuous. By duality for some $f$ strictly positive, continuous functional $f$ of $M$,

$$
\left\langle x, P_{M}^{*}(f)\right\rangle=\left\langle P_{M}(x), f\right\rangle, x \in E .
$$

Hence if we suppose that there is some $x_{0} \in E_{+} \backslash\{0\}$ such that $g\left(x_{0}\right)=P_{M}^{*}(f)\left(x_{0}\right)=1$, while $P_{M}\left(x_{0}\right)=0$. But this leads to a contradiction.

Corollary 21 If $E$ is a Banach lattice which has the Strictly Positive Martingale Property with respect to some filtration $\left(P_{a}\right)_{a \in A}$, where $A$ is a directed set. If $a \in A$ such that $P_{a} E=M_{a}$, then every strictly positive and continuous functional $f: M_{a} \rightarrow \mathbb{R}$, admits a strictly positive, continuous extension on $E$.

Corollary 22 Let $E$ be a Banach lattice of financial positions and $M$ be an incomplete market, such that $(M, f)$ is a market model. If $M$ admits a strictly positive projection, then for every price system $f: M \rightarrow \mathbb{R}$, the market model $(M, f)$ is viable.

The existence of a strictly positive projection may be replaced by the Strictly Positive Martingale Property with respect to some filtration in the statement of the above Theorem. The term viable is the one established in the seminal work of D.M. Kreps (see [3], p. 18-19).

Theorem 23 (Order 2nd Fundamental Theorem of Asset Pricing) Let $E$ be a Banach lattice and $M$ be a dense sublattice of $E$. If $M$ admits a strictly positive projection, then every strictly positive and continuous functional $f: M \rightarrow \mathbb{R}$, admits a unique strictly positive, continuous extension on $E$. Also, let $E$ be a Banach lattice and $M$ be a sublattice of $E$ such that $M$ admits a strictly positive projection. Moreover, every strictly positive and continuous functional $f: M \rightarrow \mathbb{R}$, admits a unique strictly positive, continuous extension on $E$. Then $M$ is dense in $E$.

Proof: Since $M$ is a dense sublattice of $E$, the adjoint (linear by the duality $\left\langle x, P^{*}(f)\right\rangle=\langle P(x), f\rangle, x \in E$ ) operator $P^{*}: M^{*} \rightarrow E^{*}$ of the strictly positive projection $P: E \rightarrow M$ is a surjection. Hence for any $g^{*} \in E^{*}$, there is some $h^{*} \in M^{*}$, such that $g^{*}=P^{*}\left(h_{g^{*}}^{*}\right)$, or else by duality relations: 


$$
\left\langle x, P^{*}\left(h_{g^{*}}^{*}\right)\right\rangle=\left\langle x, g^{*}\right\rangle=\left\langle P(x), h_{g^{*}}^{*}\right\rangle, x \in E .
$$

For the converse, we have that for any $g^{*} \in E^{*}$, there is some $h^{*} \in M^{*}$, such that $g^{*}=P^{*}\left(h_{g^{*}}^{*}\right)$, or else by duality relations:

$$
\left\langle x, P^{*}\left(h_{g^{*}}^{*}\right)\right\rangle=\left\langle x, g^{*}\right\rangle=\left\langle P(x), h_{g^{*}}^{*}\right\rangle, x \in E
$$

where $P: E \rightarrow M$ is a strictly positive projection. This implies that $P^{*}: M^{*} \rightarrow E^{*}$ is a surjection, which is equivalent to the fact that $M$ is dense in $E$.

Corollary 24 If $E$ is a Banach lattice which has the Strictly Positive Martingale Property with respect to some filtration $\left(P_{a}\right)_{a \in A}$, where $A$ is a directed set. If $a_{0}$ is an element of $A$ such that $P_{a_{0}} E=M_{a_{0}}$ is a dense sublattice of $E$, then every strictly positive and continuous functional $f: M_{a_{0}} \rightarrow \mathbb{R}$, admits a unique strictly positive, continuous extension on $E$.

Corollary 25 Let $E$ be a Banach lattice of financial positions and $M$ be a complete market, such that $(M, f)$ is a market model. If $M$ admits a strictly positive projection, then for every price system $f: M \rightarrow \mathbb{R}$, the market model $(M, f)$ is viable.

The term viable is the one established in the seminal work of D.M. Kreps (see [3], pp. 18-19).

We may notice that our Theorem does not make any reference to the No -Free Lunch Condition, but it simply extends the No-Arbitrage Property all over the space $E$. Theorem 23 is the analog of the usual 2nd FTAP, which implies that the (local) Equivalent Martingale Measures' set of a complete market is a singleton, while under this class of market spaces the uniqueness of the (strictly positive) extension of a price system all over the space of financial positions is achieved under no presence of the No-Free Lunch Condition, too.

Let us see some Examples which confirm the connection of the above Theorems to well-known models of Mathematical Finance.

Example 26 Let $(\Omega, \mathcal{F}, \mu)$ be a probability space endowed with an $m$-dimensional Brownian motion $B=(B(t))_{t \in[0, T]}(m \in \mathbb{N})$, where $T>0$. Denote by $\mathbb{F}=\left(\mathcal{F}_{t}\right)_{t \in[0, T]}$ the filtration that this Brownian motion generates, i.e., $\mathcal{F}_{t}=\sigma(B(u), u \in[0, t])$. We assume a financial market consisting of $n+1$ assets whose prices are modelled by an $\mathbb{F}$-adapted, $(n+1)$-dimensional Itô process $X=(X(t))_{t \in[0, T]}(n \in \mathbb{N})$ of the form $X(\cdot)=\left(X_{0}(\cdot), \cdots, X_{n}(\cdot)\right)$ where:

$$
\begin{aligned}
& \mathrm{d} X_{0}(t)=r(t) X_{0}(t) \mathrm{d} t, \\
& \mathrm{~d} X_{i}(t)=m_{i}\left(t, X_{t}\right) \mathrm{d} t+\sigma_{i}\left(t, X_{t}\right) \mathrm{d} B(t), \\
& X_{0}(0)=1, \mu-\text { a.e., } \\
& X_{i}(0)=\theta_{i}, \mu \text {-a.e., }
\end{aligned}
$$

where $\sigma_{i}(\cdot)$ is the $i$-th row $(i=1,2, \cdots, n)$ of the $n \times m$-matrix process $\sigma(\cdot)$. The process $X_{0}(\cdot)$ represents the price of a riskless asset (where $r(\cdot)$ is the interest rate process which is supposed to have bounded values), while the $i$-th component $X_{i}(\cdot)$, of the process $X(\cdot)$, represents the evolution of the price of the $i$ -th asset (stock). The price of the riskless asset may be used as numeraire. Suppose that $W \in L^{2}\left(\Omega, \mathcal{F}_{T}, \mu\right)$. If $\left(Z_{t}\right)_{t \in[0, T]}$ is a stochastic exponential, then as it is well-known, the following relation holds:

$$
\mathbb{E}_{Q}\left(W \mid \mathcal{F}_{t}\right)=\frac{1}{Z_{t}} \mathbb{E}_{\mu}\left(Z_{T} W \mid \mathcal{F}_{t}\right), \mu \text {, Q, a.e., } t<T
$$

where $Q$ is the probability measure defined on $\mathcal{F}_{T}$ as follows: $Q(A)=\int_{A} Z_{T} \mathrm{~d} \mu, A \in \mathcal{F}_{T}$, according to the Girsanov-Cameron-Martin Theorem. Taking mean values over $\mu$ we have:

$$
\mathbb{E}_{\mu}\left(W Z_{T}\right)=\mathbb{E}_{\mu}\left(\frac{1}{Z_{t}} \mathbb{E}_{\mu}\left(Z_{T} W \mid \mathcal{F}_{t}\right)\right),
$$


which in terms of evaluation maps' values is interpreted as follows:

$$
\left\langle X Z_{T}, 1\right\rangle=\left\langle\left(\mathbb{E}_{\mu}\left(Z_{T} X \mid \mathcal{F}_{t}\right)\right), \frac{1}{Z_{t}}\right\rangle .
$$

The equivalent Riesz pairs are:

$$
\left\langle L^{2}\left(\Omega, \mathcal{F}_{t}, \mu\right), L^{2}\left(\Omega, \mathcal{F}_{t}, \mu\right)\right\rangle,\left\langle L^{2}\left(\Omega, \mathcal{F}_{T}, \mu\right), L^{2}\left(\Omega, \mathcal{F}_{T}, \mu\right)\right\rangle,
$$

where the strictly positive projection $P: L^{1}\left(\Omega, \mathcal{F}_{T}, \mu\right) \rightarrow L^{1}\left(\Omega_{1} \mathcal{F}, \mu\right)$ is $P(W)=\mathbb{E}_{\mu}\left(W \mid \mathcal{F}_{t}\right)$, the strictly positive linear functional $f: L^{2}\left(\Omega, \mathcal{F}_{t}, \mu\right) \rightarrow \mathbb{R}$ is $f(W)=\left\langle W, \frac{1}{7}\right\rangle$ and its strictly positive extension $P^{*}(f): L^{2}\left(\Omega, \mathcal{F}_{T}, \mu\right) \rightarrow \mathbb{R}$ is $P^{*}(f)(W)=\langle W, 1\rangle$. This Exaftrple gives also a Hilbert space taste, due to the presence of $L^{2}$-spaces, see also [12].

Example 27

$$
\mathbb{E}_{Q}\left(X \mid \mathcal{F}_{t}\right)=\frac{1}{Z_{t}} \mathbb{E}_{\mu}\left(Z_{T} X \mid \mathcal{F}_{t}\right), \mu \text {, Q, a.e., } t<T,
$$

holds for the unique possible change of measure $Q$, if the market is complete for example in the Black-Scholes model and this arises indeprendently from the unique solution of the market-price-of-risk equation.

Finally, we may revisit the Example constructed in [4], in order to quote it.

Example 28 The actual form of the elements of the subspace $M$ of $\ell^{\infty}$ is described by the following strictly positive projection:

$$
P: \ell^{\infty} \rightarrow M,\left(x_{1}, x_{2}, x_{3}, x_{4}, \cdots\right) \mapsto\left(x_{1}, x_{2}, x_{1}, x_{2}, x_{3}, x_{4}, x_{3}, x_{4}, \cdots\right) .
$$

$M$ is a sublattice of $\ell^{\infty}$ under the usual component-wise ordering. Also, $\pi=\left(\frac{1}{2^{n}}\right)_{n \in \mathbb{N}}$, while according to Theorem 3, a strictly positive extension of $\pi$ all over $\ell^{\infty}$ exists, through duality relation $f(x)=\pi(P(x))$, $x \in \ell^{\infty}$.

\section{The Finite-State, One Period-Model Case}

We will show how the above Theorems 3, 23 are applied in finite -state space models.

Let us consider the two-date market model in which the number of states of the world is denotes by $S$, while the time-periods are denoted by 0 and 1 , respectively. We also consider an incomplete market of primitive assets whose time-period -1 payoffs are the positive, linearly independent vectors $y_{1}=x_{1}, y_{2}=x_{2}, \cdots, y_{J}=x_{J}$ of $\mathbb{R}^{S}$, whose span is denoted by $X$. We suppose that $X$ contains the riskless asset 1 , while $J<S$, which implies standard incompleteness. We also assume a time-period 0 , no-arbitrage price $q=\left(q^{1}, q^{2}, \cdots, q^{J}\right)$ for the primitive assets. As it is well-known from ([7], p. 4), $F_{1}(X)$ is identified to the sublattice $S(X)$ of $\mathbb{R}^{S}$ generated by $X$. We also remind of the following Projection Basis Theorem for sublattices of $\mathbb{R}^{s}$, which arises from both ([13], Th. 3.7), ([14], Th. 9).

Theorem 29 Let $X$ be a $J$-dimensional subspace of $\mathbb{R}^{S}$ with $J<S$ generated by the positive elements $y_{1}, y_{2}, \cdots, y_{J}$ in which the riskless bond 1 is a marketed asset $(1 \in X)$. Suppose that the range $R(\beta)$ of the basic function $\beta$ of the elements $y_{1}, y_{2}, \cdots, y_{J}$ is the finite set $\left\{P_{1}, P_{2}, \cdots, P_{\mu}\right\}$ of the simplex $\Delta_{J-1}$ of $\mathbb{R}^{J}$ (note that $\mu \geq J$ ). Suppose that the first $J$ vectors of this set are linearly independent. If we suppose that the vectors $y_{J+i}, i=1,2, \cdots, \mu-J$ are such that $y_{J+i}(s)=y(s), s \in I_{J+i}$ and $y_{J+i}(s)=0, s \notin I_{J+i}$, where $y(s)=y_{1}(s)+y_{2}(s)+\cdots+y_{J}(s)$ where $s \in \Omega$ and $I_{J+i}=\left\{s \in \Omega \mid \beta(s)=P_{J+i}\right\}, i=1,2, \cdots, \mu-J$ (which are the vectors indicated by ([13], Th. 3.7), then,

1) $S(X)=X \oplus\left[y_{J+1}, y_{J+2}, \cdots, y_{\mu}\right]$.

2) $b_{i}=2 y_{i}, i=J+1, J+2, \cdots, \mu$.

3) If $b_{i}=\tilde{b}_{i}+b_{i}^{\prime}, i=1,2, \cdots, J$ with $\tilde{b}_{i} \in X$ and $b_{i}^{\prime} \in\left[y_{J+1}, y_{J+2}, \cdots, y_{\mu}\right]$ then the vectors $\tilde{b}_{i}$ defined by: 


$$
\left(\tilde{b}_{1}, \tilde{b}_{2}, \cdots, \tilde{b}_{J}\right)^{\mathrm{T}}=A^{-1} \cdot\left(y_{1}, y_{2}, \cdots, y_{J}\right)^{\mathrm{T}}
$$

where $A$ is the $J \times J$ matrix whose columns are the vectors $P_{i}, i=1,2, \cdots, J$ are a basis of $X$ called projection basis. This basis has the property: The $J$ first coordinates of an element $x \in X$ in the positive basis of $S(X)$ coincide with the coordinates of the expansion of $x$ in the basis $\left\{\tilde{b}_{i}, i=1,2, \cdots, J\right\}$.

Also, according to what is mentioned in [7] about the completion of an incomplete market $X$ by options and by following the notation we introduced, $y=y_{1}+y_{2}+\cdots+y_{J}+y_{J+1}+\cdots+y_{\mu}$, where $\mu=\operatorname{dim} F_{1}(X)$ and $\left\{y_{1}, y_{2}, \cdots, y_{\mu}\right\}$ is a maximal set of linearly independent, positive vectors of $F_{1}(X)$. Due to ([7], Th. 21), $y_{J+1}, \cdots, y_{\mu}$ are portfolios of call and put options written on elements of $X$, especially since $1 \in X$.

The dimension equation which holds in the case of the no-arbitrage price $q$, is:

$$
\langle W\rangle \oplus\langle W\rangle^{\perp}=S+1,
$$

where $\langle W\rangle$ denotes the subspace of $\mathbb{R}^{S+1}$ generated by the columns of the payoff matrix $W(q, X)$ of the primitive securities, while $\langle W\rangle^{\perp}$ denotes the orthogonal subspace of it. Due to the characterization of the absence of arbitrage in the primitive asset market (see [15], Th. 9.2), there is at least one $\pi \in \mathbb{R}_{++}^{S+1}$ such that $\pi \cdot W(q, X)=0$ where $0 \in \mathbb{R}^{J}$. This implies that $\langle W\rangle^{\perp} \neq\{0\}$ in this case, while $q=\pi_{1} \cdot X$ if by $X$ we also denote the $S \times J$ matrix whose columns are the vectors $x_{1}, x_{2}, \cdots, x_{J}$. The last relation arises from $\pi \cdot W(q, X)=0$ if we suppose that $\pi=\left(\pi_{0}, \hat{\pi}_{1}\right)$. Then $\pi_{0} q=\hat{\pi}_{1} \cdot X$ and if we denote $\frac{1}{\pi_{0}} \hat{\pi}_{1}=\pi_{1}$, we obtain the last relation. As it is implied in [7] $F_{1}(X)$ is determined by the positive basis $\left\{b_{1}, b_{2}, \cdots, b_{\mu}\right\}$ of it.

We also have the following:

Theorem 30 Any $\pi \in \mathbb{R}_{+}^{S}$ such that $q=\pi_{1} \cdot X$ implies a no-arbitrage price $q\left(\pi_{1}\right) \in \mathbb{R}^{\mu}$ for which the price $q\left(\pi_{1}\right)(\lambda)=q\left(\pi_{1}\right) \cdot \lambda$ of the portfolio $\lambda \in \mathbb{R}^{\mu}$ or else the price of the asset $\sum_{i=1}^{\mu} \lambda_{i} y_{i}$ lying in the completion $F_{1}(X)$ to be equal to the price of the same asset under $q \in \mathbb{R}^{J}$ if $\sum_{i=1}^{\mu} \lambda_{i} y_{i} \in X$, where $y_{i}, i=1,2, \cdots, \mu$ are the vectors indicated by the Projection Basis Theorem.

Proof: Consider the vector $q\left(\pi_{1}\right)=\pi_{1} \cdot D \cdot\left(b_{1}, b_{2}, \cdots, b_{\mu}\right)^{\mathrm{T}}$. The above vector satisfies the following equalities:

$$
\left[\begin{array}{c}
\pi_{1} \cdot y_{1} \\
\pi_{1} \cdot y_{2} \\
\vdots \\
\pi_{1} \cdot y_{\mu}
\end{array}\right]^{\mathrm{T}}=\pi_{1} \cdot\left[\begin{array}{c}
y_{1} \\
y_{2} \\
\vdots \\
y_{\mu}
\end{array}\right]^{\mathrm{T}}=\left[\begin{array}{c}
q \\
\pi_{1} \cdot y_{J+1} \\
\pi_{1} \cdot y_{J+2} \\
\vdots \\
\pi_{1} \cdot y_{\mu}
\end{array}\right]^{\mathrm{T}}
$$

The definition of the vector $q\left(\pi_{1}\right)$ allows us to prove that it is a no-arbitrage price in the subspace generated by the vectors $y_{1}, y_{2}, \cdots, y_{\mu}$ which is the completion by options $F_{1}(X)$ of $X$. If for a portfolio $\lambda \in \mathbb{R}^{\mu}$ the payoff $\sum_{i=1}^{\mu} \lambda_{i} y_{i}$ lies in the positive cone $\mathbb{R}_{+}^{S}$ except $\{0\}$, then:

$$
q\left(\pi_{1}\right)(\lambda)=q\left(\pi_{1}\right) \cdot \lambda=\sum_{i=1}^{\mu} \lambda_{i}\left(\pi_{1} \cdot y_{i}\right)=\pi_{1} \cdot\left(\sum_{i=1}^{\mu} \lambda_{i} y_{i}\right)>0,
$$

because $\pi_{1} \in \mathbb{R}_{++}^{S}$. Also, from the Projection Basis Theorem 29, if $\sum_{i=1}^{\mu} \lambda_{i} y_{i} \in X$, this means that:

$$
\sum_{i=1}^{\mu} \lambda_{i} y_{i}=\sum_{i=1}^{J} \lambda_{i} y_{i} .
$$

Hence $q\left(\pi_{1}\right)(\lambda)=\sum_{i=1}^{J} \lambda_{i} \pi_{1} \cdot y_{i}$ in this case, which is equal to the valuation of the portfolio $\left(\lambda_{1}, \lambda_{2}, \cdots, \lambda_{J}\right)$ of the primitive assets under $q$. We remind that $\mathbb{R}^{\mu}$ is the space of the financial positions, since $F_{1}(X)$ is actually equal to this space according to ([7], Pr. 6).

Theorem 31 (First Order Finite Fundamental Theorem of Asset Pricing) For any subspace $X=\operatorname{span}\left[x_{1}, x_{2}, \cdots, x_{J}\right]$ of $\mathbb{R}_{+}^{S}$, where $\mu=\operatorname{dim} F_{1}(X)$ and $1 \in X$ and $x_{i} i=1,2, \cdots, J$ are linearly independent, every strictly positive linear functional of $X$ has a strictly positive extension on $F_{1}(X)=\mathbb{R}^{\mu}$. 
Proof: Every strictly positive functional $f: X \rightarrow \mathbb{R}$ defines a no -arbitrage price $q(f)$ on $X$ as follows: $q_{i}(f)=f\left(x_{i}\right), i=1,2, \cdots, J$. According to Theorem 30, $p_{1}$ for some $\pi_{1} \in \mathbb{R}_{++}^{S}$ such that $q=\pi_{1} \cdot X$ is a strictly positive extension of $f$ on $\mathbb{R}^{\mu}$, where $p_{1}(i)=\pi_{1}(i), i \in \operatorname{supp}\left(b_{j}\right), j=1,2, \cdots, \mu$, where $\operatorname{supp}\left(b_{j}\right)$ is the support of the vector $b_{j}$ of the positive basis of $F_{1}(X)$, see ([7], Th. 6).

Proposition 32 If we suppose that the vectors of the date-1 payoffs of the primitive assets $x_{1}, x_{2}, \cdots, x_{J}$ are linearly independent and $1 \in X$, then $F_{1}(X)=\mathbb{R}^{S}$, where $X=\operatorname{span}\left[x_{1}, x_{2}, \cdots, x_{J}\right]$, except a set of vectors $x_{1}, x_{2}, \cdots, x_{J}$ of Lebesgue measure zero in $\left(\mathbb{R}^{S}\right)^{J}$.

Proof: In the last part of [7], a brief proof was given about the fact that resolving markets have the property $F_{1}(X)=\mathbb{R}^{S}$. It is also well-known that resolving matrices are in general position, namely the complement of the set of them is a null-set in the vector space of the matrices $S \times J$, whose entries are real numbers. Hence the super-set of all the $S \times J$-matrices (markets), such that $1 \in X=\operatorname{span}\left[x_{1}, x_{2}, \cdots, x_{J}\right]$ where $x_{1}, x_{2}, \cdots, x_{J}$ are linearly independent and they have the property that $F_{1}(X)=\mathbb{R}^{S}$ are also in general position.

Theorem 33 (Second Order Finite Fundamental Theorem of Asset Pricing) For almost any subspace $X=\operatorname{span}\left[x_{1}, x_{2}, \cdots, x_{J}\right]$ of $\mathbb{R}_{+}^{S}$, where $S=\operatorname{dim} F_{1}(X)$ and $1 \in X$ and $x_{i} i=1,2, \cdots, J$ are linearly independent, every strictly positive linear functional of $X$ has a unique strictly positive extension on $F_{1}(X)=\mathbb{R}^{S}$.

Proof: Every strictly positive functional $f: X \rightarrow \mathbb{R}$ defines a no-arbitrage price $q(f)$ on $X$ as follows: $q_{i}(f)=f\left(x_{i}\right), i=1,2, \cdots, J$. According to Theorem 30, $q(f)=\pi_{1} \cdot X$ for a unique $\pi_{1} \in \mathbb{R}_{++}^{S}$.

\section{The Finite Multi-Period Model Case}

Let us see what happens in the multi-period framework. We consider the event -tree model as it is presented in [15], according to which there is a finite time -horizon $\mathbb{T}=\{0,1,2, \cdots, T\}$, a family of partitions $\mathrm{F}$ of $\Omega$ such that $F_{0}=\{\Omega\}, F_{T}=\{\{\omega\}, \omega=1,2, \cdots, S\}$ and $F_{t+1}$ is thinner than $F_{t}$ for any $t=0,1, \cdots, T-1$ in the sense that for any $\sigma_{t+1} \in F_{t+1}$, there is a $\sigma_{t} \in F_{t}$ such that $\sigma_{t+1} \subseteq \sigma_{t}$. Then the set $\mathbb{D}=\left\{\xi=(t, \sigma) \mid \sigma \in F_{t}, t \in \mathbb{T}\right\}$ is the event-tree corresponding to the family of partitions $\mathbb{F}$. Every event-tree $\mathbb{D}$ is a model of information revealing along the time-periods of $\mathbb{T}$. We also consider $J$ assets (financial contracts) whose payoff vectors are $V^{1}, V^{2}, \cdots, V^{J} \in \mathbb{R}^{\mathbb{D}}$ and if we denote by $n$ the physical number which is equal to the cardinality of the nodes of the event-tree $\mathbb{D}$, these are actually vectors of $\mathbb{R}^{n}$. We also suppose that the price vectors of the assets are $q^{1}, q^{2}, \cdots, q^{J}$, where $q^{j}(\xi)=0$ if $\xi \in \mathbb{D}_{T}, j=1,2, \cdots, J$ and the set $\mathbb{D}_{T}$ denotes the set of nodes of the event-tree corresponding to the time-period $T$. If we suppose that these price vectors do not provide arbitrage opportunities in the market of the assets $j=1,2, \cdots, J$, then since the market is incomplete there is at least one node-price vector $\pi \in \mathbb{R}_{++}^{n}$ such that $\pi \cdot W(q, V)=0$, where $W(q, V)$ is the payoff matrix of this market as it is indicated in ([15], Ch. 4). In order to simplify things, we may suppose that $\pi\left(\xi_{0}\right)=1$, where $\xi_{0}=(0, \Omega)$. We also suppose that one of the assets of the market is riskless, or else that for any $\xi \in \mathbb{D}$ which corresponds to the same time-period, its payoff is the same. Also, this asset's initial price $q\left(\xi_{0}\right)$ is equal to 1 . The submatrix $\left[V\left(\xi^{+}\right)+q\left(\xi^{+}\right)\right]$for any $\xi \in \mathbb{D}^{-}$is the $b(\xi) \times J$-matrix whose rows are the vectors $V\left(\xi^{\prime}\right)+q\left(\xi^{\prime}\right)$ of $\mathbb{R}^{J}$, indicating the payoffs and the ex-payoff price of the $J$ primitive securities at the node $\xi^{\prime} \in \xi^{+}$. The cardinality of $\xi^{+}, \xi \in \mathbb{D}^{-}$is denoted by $b(\xi)$.

The market of the securities is complete or as it is usually said the securities' markets are dynamically complete, if every contingent claim $c=\left(c(\xi), \xi \in \mathbb{D}^{+}\right)$can be replicated by a portfolio $z=\left(z(\xi), \xi \in \mathbb{D}^{-}\right)$, $z(\xi) \in \mathbb{R}^{J}$. In order to understand the next, we remind of the following,

Definition 34 The forward -start call option written on a contingent claim $c=\left(c(\xi), \xi \in \mathbb{D}^{+}\right)$with exerciseprice a at the node $\xi \in \mathbb{D}^{-}$given that $1_{b(\xi)} \in X(\xi)$, is equal to:

$$
\left(c\left(\xi^{+}\right)-a 1_{b(\xi)}\right)^{+} .
$$

Definition 35 The forward -start put option written on a contingent claim $c=\left(c(\xi), \xi \in \mathbb{D}^{+}\right)$with exerciseprice a at the node $\xi \in \mathbb{D}^{-}$given that $1_{b(\xi)} \in X(\xi)$, is equal to:

$$
\left(a 1_{b(\xi)}-c\left(\xi^{+}\right)\right)^{+} \text {. }
$$

As a reference for these options we append to ([16], Par. 9.2).

The market is (dynamically) complete if and only if it is one-period complete for any non-terminal node 
$\xi \in \mathbb{D}^{-}$, namely if $\operatorname{rank}\left[V\left(\xi^{+}\right)+q\left(\xi^{+}\right)\right]=b(\xi)$. Otherwise it is called incomplete. For any $\pi \in \mathbb{R}_{++}^{n}$ such that $\pi \cdot W(q, V)=0$, anc moreover there is a non-terminal node $\xi \in \mathbb{D}^{-}$such that for the corresponding submatrix $\left[V\left(\xi^{+}\right)+q\left(\xi^{+}\right)\right]$of $W(q, V)$, $\operatorname{rank}\left[V\left(\xi^{+}\right)+q\left(\xi^{+}\right)\right]<b(\xi)$ holds, we may add forward-start options of the form $\left(x-a 1_{b(\xi)}\right)^{+},\left(a 1_{b(\xi)}-x\right)^{+}$, where $x \in \operatorname{span}\left[V\left(\xi^{+}\right)+q\left(\xi^{+}\right)\right]$to make it complete. In the same way we may talk about the completion by options of the span $X(\xi)=\operatorname{span}\left[V\left(\xi^{+}\right)+q\left(\xi^{+}\right)\right]$with respect to the asset $1_{b(\xi)}$ which may be denoted by $F_{b_{b(\xi)}}(X(\xi))$ for any $\xi \in \mathbb{D}^{-} .1_{b(\xi)}$ is the vector of the Euclidean space $\mathbb{R}^{b(\xi)}$ such that $1_{b(\xi)}\left(\xi^{\prime}\right)=1, \xi^{\prime} \in \xi^{+}, \xi \in \mathbb{D}^{-}$. In a way similar to [7], the dimension of the completion $F_{b_{b(\xi)}}(X(\xi))$ is denoted by $\mu(\xi)$. It is obvious that we may reach a complete market if and only if $\mu(\xi)=b(\xi)$ for any $\xi \in \mathbb{D}^{-}$. A question which also arises in this case is how the new assets introduced in a submarket $X\left(\xi_{1}\right), \xi_{1} \in \mathbb{D}^{-}$with $\operatorname{rank}\left[V\left(\xi_{1}^{+}\right)+q\left(\xi_{1}^{+}\right)\right]<b\left(\xi_{1}\right)$ in order to reach $\mu\left(\xi_{1}\right)$ are priced. The answer is given in the next Theorem, being equivalent to Theorem 29.

Theorem 36 For any submarket $X\left(\xi_{1}\right), \xi_{1} \in \mathbb{D}^{-}$with $\operatorname{rank}\left[V\left(\xi_{1}^{+}\right)+q\left(\xi_{1}^{+}\right)\right]<b\left(\xi_{1}\right)$ and any $\pi \in \mathbb{R}_{++}^{n}$ where $\pi \cdot W(q, V)=0$, where $q=\left(q^{1}, q^{2}, \cdots, q^{J}\right)$ is a no-arbitrage price vector for the assets $i=1,2, \cdots, J$. $q(\pi)\left(\xi_{1}\right) \in \mathbb{R}^{\mu\left(\xi_{1}\right)}$ is a price vector which assigns the price $q(\pi)\left(\xi_{1}\right) \cdot \lambda$ to the portfolio $\lambda \in \mathbb{R}^{\mu\left(\xi_{1}\right)}$. Specifically, the price of the asset $\sum_{i=1}^{\mu\left(\xi_{1}\right)} \lambda_{i} y_{i}$ lying in the completion $F_{b_{\left(\xi_{1}\right)}}\left(X\left(\xi_{1}\right)\right)$ is equal to the price of the same asset under $q \in \mathbb{R}^{J}$ if $\sum_{i=1}^{\mu\left(\xi_{1}\right)} \lambda_{i} y_{i} \in X\left(\xi_{1}\right)$, where $y_{i}, i=1,2, \cdots, \mu\left(\xi_{1}\right)$ are the vectors indicated by the Projection Basis Theorem.

Proof: Consider the vector $q(\pi)\left(\xi_{1}\right)=\pi\left(\xi_{1}^{+}\right) \cdot D \cdot\left(b_{1}, b_{2}, \cdots, b_{\mu\left(\xi_{1}\right)}\right)^{\mathrm{T}}$, where $\pi\left(\xi_{1}^{+}\right) \in \mathbb{R}_{++}^{b\left(\xi_{1}\right)}$. The above vector satisfies the following equalities:

$$
\left[\begin{array}{c}
\pi\left(\xi_{1}^{+}\right) \cdot y_{1} \\
\pi\left(\xi_{1}^{+}\right) \cdot y_{2} \\
\vdots \\
\pi\left(\xi_{1}^{+}\right) \cdot y_{\mu\left(\xi_{1}\right)}
\end{array}\right]^{\mathrm{T}}=\pi\left(\xi_{1}^{+}\right) \cdot\left[\begin{array}{c}
y_{1} \\
y_{2} \\
\vdots \\
y_{\mu\left(\xi_{1}\right)}
\end{array}\right]^{\mathrm{T}}=\left[\begin{array}{c}
q\left(\xi_{1}\right) \\
\pi\left(\xi_{1}^{+}\right) \cdot y_{J+1} \\
\pi\left(\xi_{1}^{+}\right) \cdot y_{J+2} \\
\vdots \\
\pi\left(\xi_{1}^{+}\right) \cdot y_{\mu\left(\xi_{1}\right)}
\end{array}\right]^{\mathrm{T}} .
$$

The definition of the vector $q(\pi)\left(\xi_{1}\right)$ allows us to prove that it is a no-arbitrage price in the subspace generated by the vectors $y_{1}, y_{2}, \cdots, y_{\mu\left(\xi_{1}\right)}$ which is the completion by options $F_{b_{b\left(\xi_{1}\right)}}\left(X\left(\xi_{1}\right)\right)$,

$$
X\left(\xi_{1}\right)=\operatorname{span}\left[V\left(\xi_{1}^{+}\right)+q\left(\xi_{1}^{+}\right)\right] .
$$

If for a portfolio $\lambda \in \mathbb{R}^{\mu\left(\xi_{1}\right)}$ the payoff $\sum_{i=1}^{\mu\left(\xi_{1}\right)} \lambda_{i} y_{i}$ lies in the positive cone $\mathbb{R}_{+}^{b\left(\xi_{1}\right)}$ except $\{0\}$, then:

$$
q(\pi)\left(\xi_{1}\right) \cdot \lambda=\sum_{i=1}^{\mu\left(\xi_{1}\right)} \lambda_{i}\left(\pi_{1}\left(\xi_{1}^{+}\right) \cdot y_{i}\right)=\pi\left(\xi_{1}^{+}\right) \cdot\left(\sum_{i=1}^{\mu\left(\xi_{1}\right)} \lambda_{i} y_{i}\right)>0
$$

because $\pi\left(\xi_{1}^{+}\right) \in \mathbb{R}_{++}^{b\left(\xi_{1}\right)}$. Also, from the Projection Basis Theorem, if $\sum_{i=1}^{\mu\left(\xi_{1}\right)} \lambda_{i} y_{i} \in X\left(\xi_{1}\right)$, this means that $\sum_{i=1}^{\mu\left(\xi_{1}\right)} \lambda_{i} y_{i}=\sum_{i=1}^{J} \lambda_{i} y_{i}$. Hence $q(\pi)\left(\xi_{1}\right) \cdot \lambda=\sum_{i=1}^{J} \lambda_{i} \pi_{1} \cdot y_{i}$ in this case, which is equal to the valuation of the portfolio $\left(\lambda_{1}, \lambda_{2}, \cdots, \lambda_{J}\right)$ of the primitive assets under $q\left(\xi_{1}\right)$. This concludes the proof.

Theorem 37 (First Order Event-Tree Fundamental Theorem of Asset Pricing). For any submarket $X\left(\xi_{1}\right), \xi_{1} \in \mathbb{D}^{-}$with $\operatorname{rank}\left[V\left(\xi_{1}^{+}\right)+q\left(\xi_{1}^{+}\right)\right]<b\left(\xi_{1}\right)$ and any $\pi \in \mathbb{R}_{++}^{n}$ with $\pi \cdot W(q, V)=0$, every strictly positive linear functional of $X\left(\xi_{1}\right), \xi_{1} \in \mathbb{D}^{-}$has a strictly positive extension on $F_{\left.b_{(\xi)}\right)}\left(X\left(\xi_{1}\right)\right)=\mathbb{R}^{\mu\left(\xi_{1}\right)}$.

Proof: If $f\left(\xi_{1}\right): X\left(\xi_{1}\right) \rightarrow \mathbb{R}$ is a strictly positive functional of $X\left(\xi_{1}\right), \xi_{1} \in \mathbb{D}^{-}$, then this implies a no-arbitrage price $q_{f}\left(\xi_{1}\right) \in \mathbb{R}^{J^{1}}$ and since $q$ is given, $q\left(\xi_{1}\right)=q_{f}\left(\xi_{1}\right)$. The extension of $f\left(\xi_{1}\right)$ is $p_{1}\left(\xi_{1}\right)$ for some $\pi_{1} \in \mathbb{R}_{++}^{b\left(\xi_{1}\right)}$ such that $\pi_{1}\left(\xi_{1}\right) q_{f}\left(\xi_{1}\right)=\pi_{1}\left(\xi_{1}^{+}\right) \cdot X\left(\xi_{1}\right)$ is a strictly positive extension of $f$ on $\mathbb{R}^{\mu\left(\xi_{1}\right)}$, where 
$p_{1}\left(k^{\prime}\right)=\pi_{1}\left(k^{\prime}\right), k^{\prime} \in \operatorname{supp}\left(b_{j}\right), k^{\prime}=1,2, \cdots, \mu\left(\xi_{1}\right)$, where $\operatorname{supp}\left(b_{j}\right)$ is the support of the vector $b_{j}$ of the positive basis of $F_{1_{b\left(\xi_{1}\right)}}\left(X\left(\xi_{1}\right)\right)$, see ([7], Th. 6).

Theorem 38 (Second Order Finite Fundamental Theorem of Asset Pricing) If the market is complete, then for any submarket $X\left(\xi_{1}\right), \xi_{1} \in \mathbb{D}^{-}$, every strictly positive linear functional of $X\left(\xi_{1}\right), \xi_{1} \in \mathbb{D}^{-}$has a unique strictly positive extension on $F_{\left.1_{b(\xi)}\right)}\left(X\left(\xi_{1}\right)\right)=\mathbb{R}^{b\left(\xi_{1}\right)}$.

Proof: Since the market is complete, $\left[V\left(\xi_{1}^{+}\right)+q\left(\xi_{1}^{+}\right)\right]=b\left(\xi_{1}\right), \xi_{1} \in \mathbb{D}^{-}$and there is a unique $\pi \in \mathbb{R}_{++}^{n}$ with $\pi \cdot W(q, V)=0$, If $f\left(\xi_{1}\right): X\left(\xi_{1}\right) \rightarrow \mathbb{R}$ is a strictly positive functional of $X\left(\xi_{1}\right), \xi_{1} \in \mathbb{D}^{-}$, then this implies a no-arbitrage price $q_{f}\left(\xi_{1}\right) \in \mathbb{R}^{J}$ and since $q$ is given, $q\left(\xi_{1}\right)=q_{f}\left(\xi_{1}\right)$. The unique extension of $f\left(\xi_{1}\right)$ is $p_{1}\left(\xi_{1}\right)$ for some $\pi_{1} \in \mathbb{R}_{++}^{b\left(\xi_{1}\right)}$ such that $\pi_{1}\left(\xi_{1}\right) q_{f}\left(\xi_{1}\right)=\pi_{1}\left(\xi_{1}^{+}\right) \cdot X\left(\xi_{1}\right)$ is a strictly positive extension of $f$ on $\mathbb{R}^{b\left(\xi_{1}\right)}$, where $p_{1}\left(k^{\prime}\right)=\pi_{1}\left(k^{\prime}\right), k^{\prime} \in \operatorname{supp}\left(b_{j}\right), k^{\prime}=1,2, \cdots, b\left(\xi_{1}\right)$, where $\operatorname{supp}\left(b_{j}\right)$ is the support of the vector $b_{j}$ of the positive basis of $F_{b_{b\left(\xi_{1}\right)}}\left(X\left(\xi_{1}\right)\right)$, see ([7], Th. 6), since $\pi_{1}$ is unique.

\section{General Cones Revisited}

Let us consider a Banach space $E$ of financial positions, partially ordered by a closed cone $C$, which is not a lattice cone. Such a cone is for example a Bishop-Phepls cone, see ([17], pp. 126-127), which is well-based and it has also interior points, hence it is not a lattice cone, according to ([17], Th. 4.4.4). Of course, the set of strictly positive functionals of such a cone has not to be empty. This is the reason due to which the Lindelöf Property mentioned in [9] about the weak topology $\sigma(X, Y)$ defined on a dual system $\langle X, Y\rangle$ is important. Of course, there are cones which do not admit continuous strictly positive functionals. Such a cone is the positive cone of an $\ell^{p}(\Gamma), 1<p<\infty$ space, where $\Gamma$ is uncountable.

Also, in this section, the definition of (in)completeness are altered.

Definition 39 If $M$ is a infinite-dimensional subspace of $E$ ordered by the cone $C$, a market is an infinitedimensional subspace of $E$, such that $M=C-C$.

Definition 40 A market is incomplete if $\bar{M} \neq E$, while it is complete if $\bar{M}=E$.

Then, the following versions of the Second and the First Fundamental Theorem of Asset Pricing are deduced, respectively.

Theorem 41 Let $E$ be a Banach space with an unconditional basis. Then a non-lattice one exists, which makes $E$ a complete market and every strictly positive functional of this cone admits a unique strictly positive extension.

Proof: As it is well-known from ([18], Th. 4.2.22), the cone of the unconditional basis $\left(x_{n}\right)_{n \in \mathbb{N}}$ $K=\left\{x \in E \mid x=\sum_{n=1}^{\infty} a_{n} x_{n}, a_{n} \geq 0\right\}$ makes $X$ a Banach lattice under an equivalent norm. According to ([8], Th. 5.7) there is a strongly reflexive cone (see [8], Def. 5.1) $C$ in $E_{+}$, such that $E=\overline{C-C}$. Also, since the one-dimensional-subspace projections $P_{n}, n \in \mathbb{N}$ are continuous, according to ([18], Cor. 4.2.26), the operator $P=\sum_{n=1}^{\infty} \frac{1}{2^{n}} P_{n}$ is a continuous projection from $E$ ordered by $E_{+}$(which is also the cone of the positive basis) to $C-C$ being ordered by $C$. Also, we notice that $P$ is strictly positive in the sense that $P x=0 \Leftrightarrow x=0$, whenever $x \in E_{+}$. Hence, $P$ may be taken as a strictly positive projection, and consequently we may repeat the proof of Theorem 23.

Theorem 42 Let $E$ be a Banach space with an unconditional basic sequence. Then, for the incomplete market $Y$ arising from the basic sequence, there exists a non-lattice cone $C$, such that $Y=\overline{C-C}$ and strictly positive functional of this cone admits a strictly positive extension on $E$.

Proof: According to ([8], Cor. 5.8) there is a strongly reflexive cone (see [8], Def. 5.1) $C$ in $E_{+}$, such that $Y=\overline{C-C}$, while for any uncoditional basic sequence it is well-known that (see [18], Th. 4.2.22) its cone $Y_{+}$ makes $Y$ a Banach lattice (under an equivalent norm). Also, since the one-dimensional-subspace projections $P_{n}, n \in \mathbb{N}$ are continuous, according to ([18], Cor. 4.2.26) the operator $P=\sum_{n=1}^{\infty} \frac{1}{2^{n}} P_{n}$ is a continuous projection from $E$ ordered by $E_{+}$(which is also the cone of the positive basis) to $C-C$ being ordered by 
$C$. Also, we notice that $P$ is strictly positive in the sense that $P x=0 \Leftrightarrow x=0$, whenever $x \in E_{+}$. Hence, $P$ may be taken as a strictly positive projection, and consequently we may repeat the proof of Theorem 3 .

In the proof of ([8], Th. 5.7) the strongly reflexive cone's construction relies exactly on the existence of an unconditional basis for the Banach space $E$. Then we may understand that the crucial point for the above Theorems is the existence of a basic sequence for the Banach space $E$. We may remind the seminal work by Bessaga-Pelczynski [19] essentials on this topic.

\section{References}

[1] Delbaen, F. and Schachermayer, W. (1994) A General Version of the Fundamental Theorem of Asset Pricing. Mathematische Annalen, 300, 463-520. http://dx.doi.org/10.1007/BF01450498

[2] Delbaen, F. and Schachermayer, W. (1998) The Fundamental Theorem of Asset Pricing for Unbounded Stochastic Processes. Mathematische Annalen, 312, 215-250. http://dx.doi.org/10.1007/s002080050220

[3] Kreps, D.M. (1981) Arbitrage and Equilirium in Economies with Infinitely Many Commodities. Journal of Mathematical Economics, 8, 15-35. http://dx.doi.org/10.1016/0304-4068(81)90010-0

[4] Schachermayer, W. (2002) No Arbitrage: On the Work of David Kreps. Positivity, 6, 359-368. http://dx.doi.org/10.1023/A:1020262419556

[5] Acciaio, B., Beiglböck, M., Penkner, F. and Schachermayer, W. (2013) A Model-Free Version of the Fundamental Theorem of Asset Pricing and the Super-Replication Theorem. Mathematical Finance (to Appear), 6 December 2013. http://dx.doi.org/10.1111/mafi.12060

[6] Troitsky, V.G. (2005) Martingales in Banach lattices. Positivity, 9, 437-456. http://dx.doi.org/10.1007/s11117-004-2769-1

[7] Kountzakis, C. and Polyrakis, I.A. (2006) The Completion of Security Markets. Decisions in Economics and Finance, 29, 1-21. http://dx.doi.org/10.1007/s10203-006-0059-z

[8] Casini, E., Miglierina, E., Polyrakis, I.A. and Xanthos, F. (2013) Reflexive Cones. Positivity, 17, 911-933. http://dx.doi.org/10.1007/s11117-012-0212-6

[9] Rokhlin, D.B. (2005) The Kreps-Yan Theorem for $\ell^{\infty}$. International Journal of Mathematics and Mathematical Sciences, 17, 2749-2756.

[10] Rokhlin, D.B. (2009) The Kreps-Yan Theorem for Ideal Banach Spaces. Siberian Mathematical Journal, 50, $162-166$.

[11] Polyrakis, I.A. (1996) Finite-Dimensional Lattice-Subspaces of $C(\Omega)$ and Curves of $\mathbb{R}^{n}$. Transactions of the American Mathematical Society, 348, 2793-2810. http://dx.doi.org/10.1090/S0002-9947-96-01639-X

[12] Schachermayer, W. (1992) A Hilbert Space Proof of the Fundamental Theorem of Asset Pricing in Finite Discrete Time. Insurance: Mathematics and Economics, 11, 249-257. http://dx.doi.org/10.1016/0167-6687(92)90013-2

[13] Polyrakis, I.A. (1999) Minimal Lattice Subspaces. Transactions of the American Mathematical Society, 351, 41834203.

[14] Polyrakis, I.A. (2003) Linear Optimization in $C(\Omega)$ and Portfolio Insurance. Optimization, 52, 221-239.

[15] Magill, M. and Quinzii, M. (1996) Theory of Incomplete Markets. MIT Press, location.

[16] Musiela, M. and Rutkowski, M. (1997) Martingale Methods in Financial Modelling. Applications of Mathematics, Mathematical Modelling and Applied Probability, Springer, Berlin. http://dx.doi.org/10.1007/978-3-662-22132-7

[17] Jameson, G. (1970) Ordered Linear Spaces. Lecture Notes in Mathematics, Springer-Verlag, Berlin.

[18] Megginson, R.E. (1998) An Introduction to Banach Spaces. Springer, New York. http://dx.doi.org/10.1007/978-1-4612-0603-3

[19] Bessaga, C. and Pełczyński, A. (1958) On Bases and Unconditional convergence of Series in Banach Spaces. Studia Mathematica, 17, 151-164.

[20] Aliprantis, C.D. and Border, K.C. (2005) Infinite Dimensional Analysis, A Hitchhiker’s Guide. 3rd Edition, Springer, Berlin. 


\section{Appendix}

In this Section, we give some essential notions and results from the theory of partially ordered linear spaces which are used in this paper. For these notions and definitions, see ([17], Ch. 1, Ch. 2, Ch. 3). Let $E$ be a (normed) linear space. A set $C \subseteq E$ satisfying $C+C \subseteq C$ and $\lambda C \subseteq C$ for any $\lambda \in \mathbb{R}_{+}$is called wedge. A wedge for which $C \cap(-C)=\{0\}$ is called cone. A pair $(E, \geq)$ where $E$ is a linear space and $\geq$ is a binary relation on $E$ satisfying the following properties:

1) $x \geq x$ for any $x \in E$ (reflexive);

2) If $x \geq y$ and $y \geq z$ then $x \geq z$, where $x, y, z \in E$ (transitive);

3) If $x \geq y$ then $\lambda x \geq \lambda y$ for any $\lambda \in \mathbb{R}_{+}$and $x+z \geq y+z$ for any $z \in E$, where $x, y \in E$ (compatible with the linear structure of $E$ ), is called partially ordered linear space. The binary relation $\geq$ in this case is a partial ordering on $E$. The set $P=\{x \in E \mid x \geq 0\}$ is called (positive) wedge of the partial ordering $\geq$ of $E$. Given a wedge $C$ in $E$, the binary relation $\geq_{C}$ defined as follows:

$$
x \geq_{C} y \Leftrightarrow x-y \in C,
$$

is a partial ordering on $E$, called partial ordering induced by $C$ on $E$. If the partial ordering $\geq$ of the space $E$ is antisymmetric, namely if $x \geq y$ and $y \geq x$ implies $x=y$, where $x, y \in E$, then $P$ is a cone.

$E^{\prime}$ denotes the linear space of all linear functionals of $E$, called algebraic dual while $E^{*}$ is the norm dual of $E$, in case where $E$ is a normed linear space.

Suppose that $C$ is a wedge of $E$. A functional $f \in E^{\prime}$ is called positive functional of $C$ if $f(x) \geq 0$ for any $x \in C . f \in E^{\prime}$ is a strictly positive functional of $C$ if $f(x)>0$ for any $x \in C \backslash\{0\}$. A linear functional $f \in E^{\prime}$ where $E$ is a normed linear space, is called uniformly monotonic functional of $C$ if there is some real number $a>0$ such that $f(x) \geq a\|x\|$ for any $x \in C$. In case where a uniformly monotonic functional of $C$ exists, $C$ is a cone. $C^{0}=\left\{f \in E^{*} \mid f(x) \geq 0\right.$ for any $\left.x \in C\right\}$ is the dual wedge of $C$ in $E^{*}$. Also, by $C^{00}$ we denote the subset $\left(C^{0}\right)^{0}$ of $E^{* *}$. It can be easily proved that if $C$ is a closed wedge of a reflexive space, then $C^{00}=C$. If $C$ is a wedge of $E^{*}$, then the set $C_{0}=\{x \in E \mid \hat{x}(f) \geq 0$ for any $f \in C\}$ is the dual wedge of $C$ in $E$, where $\hat{:} E \rightarrow E^{* *}$ denotes the natural embedding map from $E$ to the second dual space $E^{* *}$ of $E$. Note that if for two wedges $K, C$ of $E, K \subseteq C$ holds, then $C^{0} \subseteq K^{0}$.

If $C$ is a cone, then a set $B \subseteq C$ is called base of $C$ if for any $x \in C \backslash\{0\}$ there exists a unique $\lambda_{x}>0$ such that $\lambda_{x} x \in B$. The set $B_{f}=\{x \in C \mid f(x)=1\}$ where $f$ is a strictly positive functional of $C$ is the base of $C$ defined by $f . B_{f}$ is bounded if and only if $f$ is uniformly monotonic. If $B$ is a bounded base of $C$ such that $0 \notin \bar{B}$ then $C$ is called well-based. If $C$ is well-based, then a bounded base of $C$ defined by a $g \in E^{*}$ exists. If $E=C-C$ then the wedge $C$ is called generating, while if $E=\overline{C-C}$ it is called almost generating. If $C$ is generating, then $C^{0}$ is a cone of $E^{*}$ in case where $E$ is a normed linear space. Also, $f \in E^{*}$ is a uniformly monotonic functional of $C$ if and only if $f \in \operatorname{int} C^{0}$, where $\operatorname{int} C^{0}$ denotes the norm-interior of $C^{0}$. If $E$ is partially ordered by $C$, then any set of the form $f \in[x, y]=\left\{r \in E \mid y \geq_{C} r \geq_{C} x\right\}$ where $x, y \in C$ is called order-interval of $E$. If $E$ is partially ordered by $C$ and for some $e \in E, E=\cup_{n=1}^{\infty}[-n e, n e]$ holds, then $e$ is called order-unit of $E$. If $E$ is a normed linear space, then if every interior point of $C$ is an order-unit of $E$. If $E$ is moreover a Banach space and $C$ is closed, then every order-unit of $E$ is an interior point of $C$. The partially ordered vector space $E$ is a vector lattice if for any $x, y \in E$, the supremum and the infimum of $\{x, y\}$ with respect to the partial ordering defined by $P$ exist in $E$. In this case $\sup \{x, y\}$ and $\inf \{x, y\}$ are denoted by $x \vee y, x \wedge y$ respectively. If so, $|x|=\operatorname{osup}\{x,-x\}$ is the absolute value of $x$ and if $E$ is also a normed space such that $\|x\|=\|x\|$ for any $x \in E$, then $E$ is called normed lattice. If a normed lattice is a Banach space, then it is called Banach lattice. A Banach lattice $E$ whose norm has the property $\|x+y\|=\|x\|+\|y\|, x, y \in E_{+}$is called $A L$-space. A set $S$ in a vector lattice $E$ is called solid if $|y| \leq|x|$ and $x \in S$ implies $y \in S$. A solid vector subspace of a vector lattice is called ideal. An ideal $I$ is a sublattice of $E$, i.e., a subspace of $E$ such that $x \vee y \in I, x \wedge y \in I$ if $x, y \in I$ respectively. A net $\left\{x_{a}\right\}_{a \in A}$ in a vector lattice $E$ is order convergent to $x$ if there is a net $\left\{y_{a}\right\}_{a \in A}$ in $E$ with $y_{a} \downarrow 0$, such that $\left|x_{a}-x\right| \leq y_{a}$ for each $a \in A$. This convergence is denoted by $x_{a} \stackrel{o}{\longrightarrow} x$. A set $D$ in $E$ is order closed if $\left\{x_{a}\right\}_{a \in A} \subseteq D$ and $x_{a} \stackrel{o}{\longrightarrow} x$, implies $x \in D$. If $D$ is also an ideal, then $D$ is called band. A Banach lattice has order continuous norm, if for any net $\left\{x_{a}\right\}_{a \in A} \subseteq E$ with $x_{a} \downarrow 0,\left\|x_{a}\right\| \downarrow 0$ holds. A Banach lattice $E$ which is a band in its second dual (in the sense of norm topology) 
is called Kantorovich-Banach space. If $S$ is a subset of a vector lattice $E$, then its disjoint complement is the set $S^{d}=\{x \in E:|x| \wedge|y|$ for any $y \in S\}$. If for a vector lattice $E$ a band $B$ satisfies the property $E=B \oplus B^{d}$, then $B$ is called projection band. Finally, if $E$ is a partially ordered Banach space whose positive cone is $E_{+}$, if $E$ has a Schauder basis $\left(e_{n}\right)_{n \in \mathbb{N}}$, this basis is called positive basis if and only if $E_{+}=\left\{x=\sum_{n=1}^{\infty} \lambda_{n} e_{n} \mid \lambda_{n} \geq 0, n \in \mathbb{N}\right\}$. For linear lattices and positive bases see in ([20], Ch. 8), and [11], respectively. 
Scientific Research Publishing (SCIRP) is one of the largest Open Access journal publishers. It is currently publishing more than 200 open access, online, peer-reviewed journals covering a wide range of academic disciplines. SCIRP serves the worldwide academic communities and contributes to the progress and application of science with its publication.

Other selected journals from SCIRP are listed as below. Submit your manuscript to us via either submit@scirp.org or Online Submission Portal.
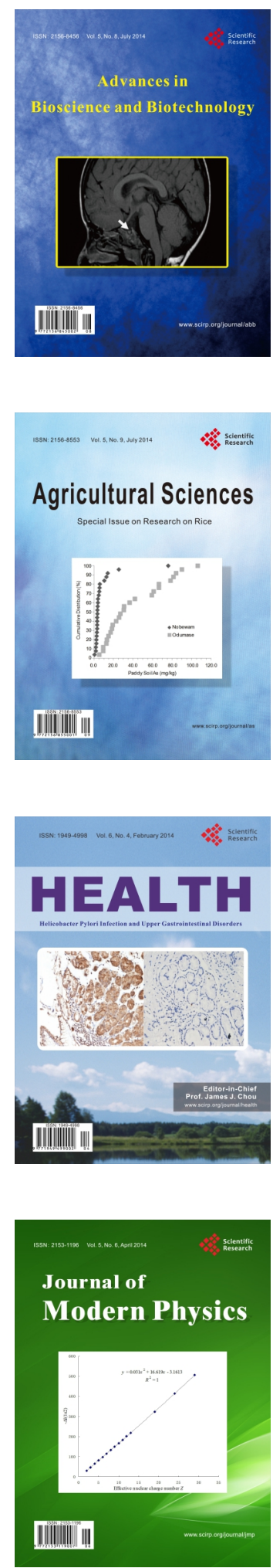
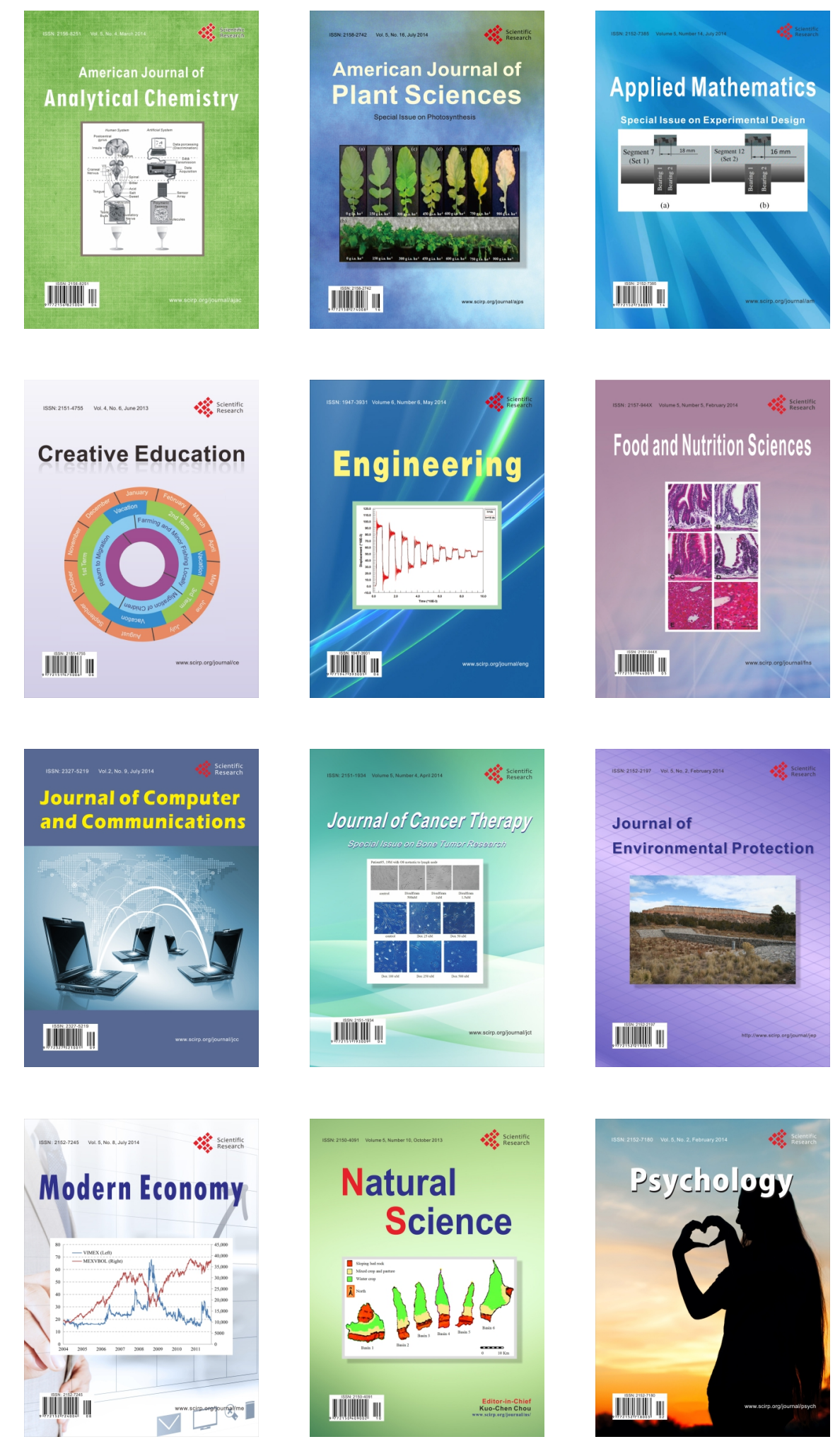